\title{
Serious Adverse Drug Events Reported to the FDA: Analysis of the FDA Adverse Event Reporting System 2006-2014 Database
}

\author{
Kalyani B. Sonawane, PhD; Ning Cheng, MS; and Richard A. Hansen, PhD
}

\begin{abstract}
BACKGROUND: Data on adverse drug events (ADEs) observed at the population level provide important evidence regarding the safety of a pharmaceutical product in real-world settings. Recent patterns in serious and fatal $A D E$ reporting have not been documented.
\end{abstract}

OBJECTIVE: To assess recent patterns in serious and fatal ADE reports in the United States.

METHODS: We conducted a retrospective analysis of the publicly available 2006-2014 FDA Adverse Event Reporting System database. Non-U.S. reports, reports from clinical trials, and reports with missing outcome data were excluded. The annual numbers of ADEs with reported outcome of death, disability, and other serious outcomes were determined. Types (direct, manufacturer expedited, or manufacturer periodic) and sources (consumer, health professional, or other) of these serious ADE reports were also identified. The distribution of serious ADE reports by patient age groups $(<18,18-44,45-64$, and $\geq 65$ years) was determined. Drugs listed as primary suspects in serious ADEs (death, disability, and other serious outcomes) were identified and ranked. Descriptive statistics were used to characterize the patterns in serious or fatal ADE reporting.

RESULTS: From 2006 to 2014, the number of serious ADEs reported to the FDA increased 2-fold. A total of 902,323 serious outcomes were reported over the 9-year study period: 244,408 deaths, 72,141 disabilities, and 585,774 other serious outcomes. The relative percentage of reports of deaths was highest during 2012 (32.4\%). The percentage of reports of disability was highest during 2006 (12.1\%). Overall, the "other serious outcomes" category accounted for almost $65 \%$ of serious ADEs reports. Expedited reports from drug manufacturers were most common (about $72 \%$ ) of the serious ADEs with available data on report type. Health professionals $(47.3 \%)$ were the most common source of report followed by consumers $(36.1 \%)$ and other sources (16.6\%). A disproportionately high number of reported ADEs was among patients aged $45-64$ years $(40 \%)$ and $\geq 65$ years (32.6\%). Antineoplastic drugs were more frequently reported with deaths. Three antidepressant drugs were among the top 10 drugs reported with disability. During 2006-2014, there were 38 drugs with more than 1,000 reports of serious ADEs in a given year: 2 drugs currently withdrawn from the market (rofecoxib and parecoxib), 10 drugs with an FDA risk evaluation and mitigation strategies (REMS) program, 13 biologic or specialty drugs, and 14 others.

CONCLUSIONS: An overall increase in the trend of the number of serious ADE reports was observed from 2006 to 2014. Drugs with a REMS program and biologic and specialty drugs were involved in a significant number of reported serious ADEs. Data on reporting patterns can guide surveillance and pharmacoepidemiological studies to understand the public health burden of serious ADEs.

J Manag Care Spec Pharm. 2018;24(7):682-90

Copyright $\odot 2018$, Academy of Managed Care Pharmacy. All rights reserved.

\section{What is already known about this subject}

Each year more than 770,000 people are injured or die from adverse drug events (ADEs).

According to a previous study, from 1998 to 2005, there was a 2.6-fold increase in the reports of serious ADEs.

\section{What this study adds}

A 2-fold increase in serious ADE reports was observed from 2006 through 2014, which was similar to a previously reported increasing trend in serious ADE reports from 1998 to 2005.

This study found that 13 biologic or specialty drugs were connected with 1,000 reports of serious ADEs in a given year during 2006-2015.

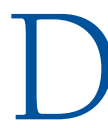
uring the past decade, an average of 75 new drug applications have been approved annually by the U.S. Food and Drug Administration (FDA). ${ }^{1}$ Although these drugs were approved for use after established safety and efficacy from clinical trials, postmarketing surveillance is necessary to ensure their continued safety once these drugs reach the general population. Data on anticipated and unanticipated adverse drug events (ADEs; i.e., injuries resulting from the use of the drug) observed at the population level provide important evidence regarding the safety of a drug in real-world settings.

To understand the postmarketing safety of approved drugs, a system to monitor ADEs was initiated by the FDA in 1969. In 1998, the system was redesigned and a spontaneous ADE reporting database - the FDA Adverse Event Reporting System (FAERS, formerly AERS) — was launched. ${ }^{2}$ The database is used by the FDA and health care researchers to study safety-related issues for drugs.

According to FDA statistics, the number of ADEs reported to FAERS resulting in death and serious outcomes increased consistently from 2006 through 2014. ${ }^{3}$ A previously published study by Moore et al. (2007) found that from 1998 to 2005, there was a 2.6-fold increase in the reports of serious ADEs and a 2.7fold increase in the reports of fatal ADEs. ${ }^{4}$ About 80,880 deaths were reported during this period, along with 32,922 and 354,007 reports of disabilities and other serious outcomes, respectively. ${ }^{4}$

The objective of this study was to update the data presented by Moore et al., in order to establish more current documentation 
of patterns in FAERS reports of serious ADEs and identify the drugs for which serious ADEs are more commonly reported.

\section{Methods}

\section{Database and Variables}

We conducted a retrospective analysis of the FAERS database. FAERS consists of spontaneous reports of ADEs and medication errors reported to the FDA. The dataset is publicly available on the FDA website and consists of multiple files that can be linked using unique event identifiers. ${ }^{5}$

Data files for 2006 through 2014 were used for this study. Non-U.S. reports were excluded to reduce the impact of geographic variations in ADE reporting. Reports from clinical trials were excluded due to mandatory reporting requirements. Only those reports with complete information on the outcome were retained in the final analyses. Since FAERS data may contain more than 1 report per case when follow-up information is submitted, only the most recent report for a given case was included. In order to compare the results of our study with previously published patterns of reporting, the inclusion/exclusion criteria, variable identification process, and data analysis for this study were based on previously published work by Moore et al. ${ }^{4}$

The type of report was classified as (a) direct (i.e., reports submitted directly to the FDA from consumers or health care professionals), (b) manufacturer expedited (i.e., reports from the manufacturer submitted to the FDA within 15 days of a serious and unexpected ADE not included in product labeling), ${ }^{6}$ and (c) manufacturer periodic (i.e., ADE reports from manufacturers that are already included in the product label and submitted quarterly or annually). The initial sources of reports were categorized into consumer, health professional, and other (including distributor, company representative, user facility, literature, and other sources). Four age groups $(<18,18-44,45-64$, and $\geq 65$ years) were created using patient birth dates to understand the age-wise distribution of reports. To provide context for the ADE reporting patterns, U.S. prescription drug use trends were obtained from a prescription drug use report by the Centers for Disease Control and Prevention (CDC). ${ }^{7}$

\section{Outcome and Drug Identification Process}

Outcomes in the ADE reports were recoded and each report was assigned to one of 3 hierarchical categories: death > disability (disability or congenital anomaly) $>$ or all other serious outcomes (hospitalizations, requiring intervention, life-threatening, or other serious outcomes). Drugs were identified by brand name or generic name and were recoded to the active ingredient name to determine the final count of the suspect drug. Only drugs listed as a primary suspect were included in the analysis. Drugs listed as secondary suspect were excluded due to the uncertainty of the association between the drug and the ADE. Reports for medical devices, vaccines, dietary supplements, and illegal drugs were excluded.
We identified and ranked drugs that were most commonly reported as primary suspect in serious ADE reports. A list of top 10 drugs was identified for death, disability, and other serious outcomes. We also identified the drugs with more than 1,000 ADE reports in any study year. We classified specific types of agents that could be expected to have high rates of ADEs, including drugs with an FDA risk evaluation and mitigation strategies (REMS) program, as well as biologic and specialty drugs. For drugs under REMS, the manufacturers are required to ensure that the benefits of drugs or biological product outweigh their risk through ongoing monitoring. ${ }^{8}$ Biologic and specialty drugs were explored because they introduce new, potent mechanisms of action, but also new unexpected toxicities. ${ }^{9,10}$ Also, the economic burden of specialty drugs is high; about a quarter of the total drug expenditures in the United States is accounted for by these drugs. ${ }^{11}$ We identified specialty drugs using the BlueCross-BlueShield of North Carolina specialty drug list. ${ }^{12}$

\section{Statistical Analysis}

Descriptive statistics were used to summarize data. The annual number of reports of serious ADEs was determined from 2006 through 2014. Data from a previous study were used to determine the trend of reports of serious ADEs before $2006 .{ }^{4}$ Frequencies were determined according to each outcome category or drug. The proportion of ADE reports by age group was estimated based on the total number of serious ADE reports and compared against the proportion of drug use attributable to each age group. ${ }^{7}$ All analyses were conducted using SAS software package 9.4 (SAS Institute, Cary, NC).

\section{Results}

A total of 902,323 serious outcomes were reported over the 9-year study period: 244,408 deaths, 72,141 disabilities, and 585,774 other serious outcomes. Figure 1 illustrates the trend of serious ADE reporting to FAERS over time. Overall, there was an upward trend in serious ADE reporting. From 2006 through 2014, a 2-fold increase in serious ADEs was reported to the FDA, although the reports of serious ADEs decreased from 2006 to 2007 and 2012 to 2013

The annual numbers of reports of serious ADEs from 2006 to 2014 are presented in Table 1 by outcome categories. The annual number of reports with fatal outcomes increased steadily from 2007 to 2012 but decreased during 2013 and 2014. The relative percentage of reports for deaths was highest during 2012 (32.4\%). The percentage of reports of disability was highest during 2006 (12.1\%) and remained below 10\% throughout the remaining periods. The percentage of reports of other serious outcomes was highest during 2007 (71.7\%). The number of reports of other serious outcomes increased during later years but the proportion declined. Overall, the other serious outcomes category accounted for $64.9 \%$ of serious ADEs reports. 


\section{FIGURE 1 Serious Adverse Drug Events Reported to the FDA, 1998-2014}

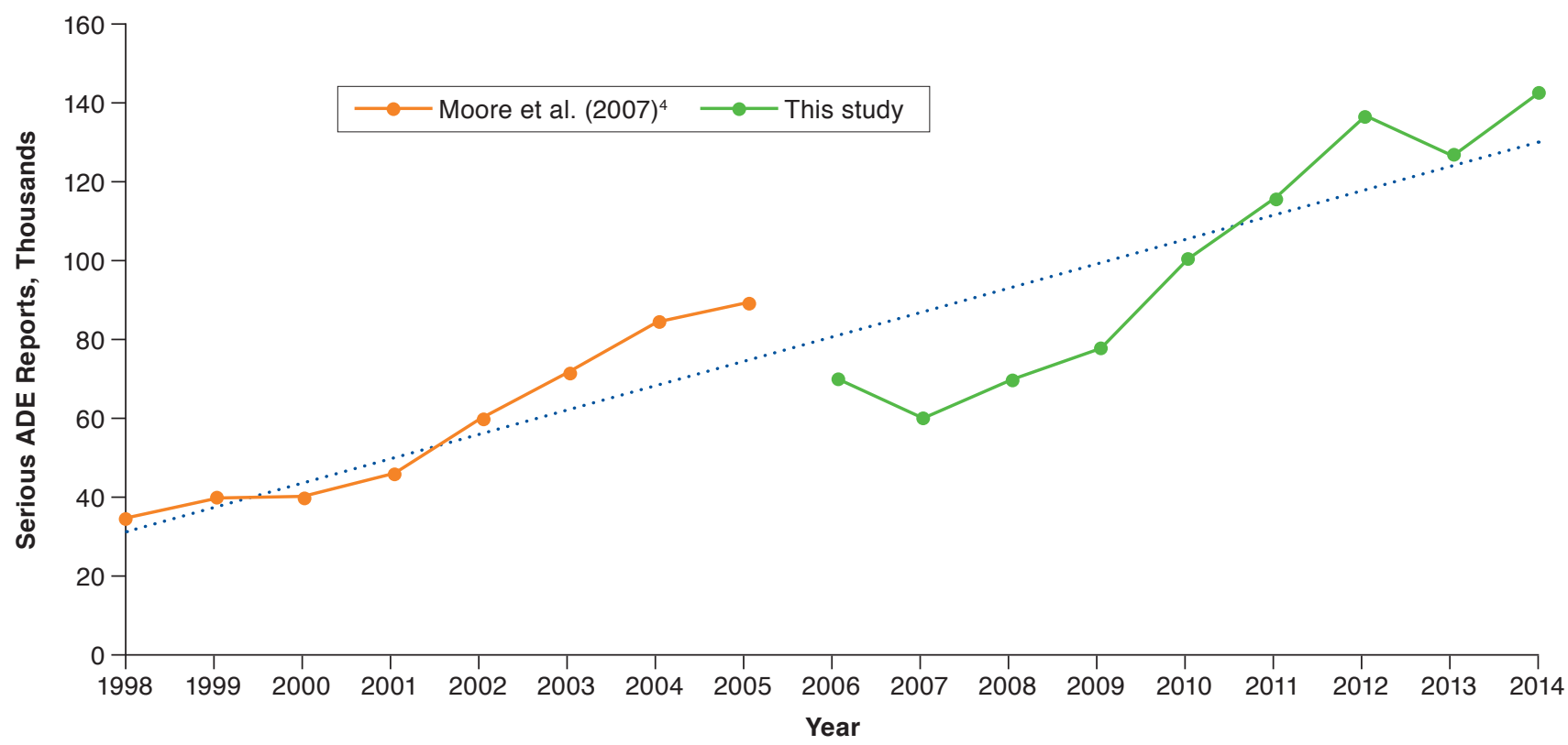

Note: This figure illustrates trends in serious ADE reporting in the United States, with death, disability, or other serious outcomes from 1998 to 2014 . Data from 1998 to 2005 were obtained from Moore et al. (2007). ${ }^{4}$ Data from 2006 to 2014 are from this study. The dotted line indicates the overall serious ADE reporting trend. $A D E=$ adverse drug event; FDA =U.S. Food and Drug Administration.

The types and sources of the serious ADEs reports are summarized in Table 1. Expedited reports from drug manufacturers were most common, accounting for about $72 \%$ of the serious ADEs with available data on report type. Only $13.6 \%$ of serious ADEs were periodic reports from the manufacturer, and $14.5 \%$ were direct reports to the FDA. Among the 186,552 reports with available data on source, health professionals (47.3\%) were the most common source of report, followed by consumers (36.1\%). Other sources accounted for $16.6 \%$ of reports.

Data on patients' ages were available for 634,285 ADEs reports. The distribution of serious ADEs reports by age is presented in Figure 2. These are compared with prescription drug use data (2007-2010) from the CDC. ${ }^{7}$ Prescription drug use was highest among those aged $18-44$ years, accounting for $32.1 \%$ of the total U.S. population using prescription drugs, while only $22 \%$ of the total serious reported ADEs occurred among this group. Serious ADEs were highest among those aged 45-64 years (40\%), while this group comprised roughly $31 \%$ of the population using prescription drugs.

Drugs with the highest number of reports of death, disability, and other serious outcomes during 2006-2014 were determined, and the 10 drugs with the highest number of reports of these serious ADEs are listed in Table 2. Three antineoplastic drugs were among the top 10 drugs with reports of deaths. Three antidepressant drugs (paroxetine, fluoxetine, and sertraline) were among the top 10 drugs reported with disability. Listed among these 10 drugs was rofecoxib, a nonsteroidal antiinflammatory drug, which was later withdrawn from the market. Lenalidomide was the only drug that was listed in the top 10 most commonly reported drugs under all 3 serious outcome categories (i.e., death, disability, and other serious outcomes).

We also identified the drugs that had $>1,000$ reports of serious ADEs during any year in the 2006-2014 FAERS data (Table 3). Of the 38 drugs identified, 2 drugs (rofecoxib and parecoxib) were withdrawn from the market, and 10 drugs had a REMS program. Thirteen drugs were identified as biologic or specialty drugs.

\section{Discussion}

$\overline{\text { Our study reported recent patterns of serious ADEs reported to }}$ the FDA-death, disability, and other serious outcomes-and demonstrates that the number of serious ADE reports doubled from 2006 to 2014. Moore et al., previously reported that the number of serious ADE reports increased by more than 2-fold from 1998 to $2005 .{ }^{4}$ Our analysis demonstrated that the reports of serious ADEs continued to increase, doubling over the 9-year study period, and reached 100,000 reports per year beginning in 2010. We noted a slight dip in the reports during 2007 and 2013 in our analysis compared with Moore et al. ${ }^{4}$ 
Serious Adverse Drug Events Reported to the FDA: Analysis of the FDA Adverse Event Reporting System 2006-2014 Database

TABLE 1 Type and Source of Serious Adverse Drug Events Reported to the FDA, 2006-2014

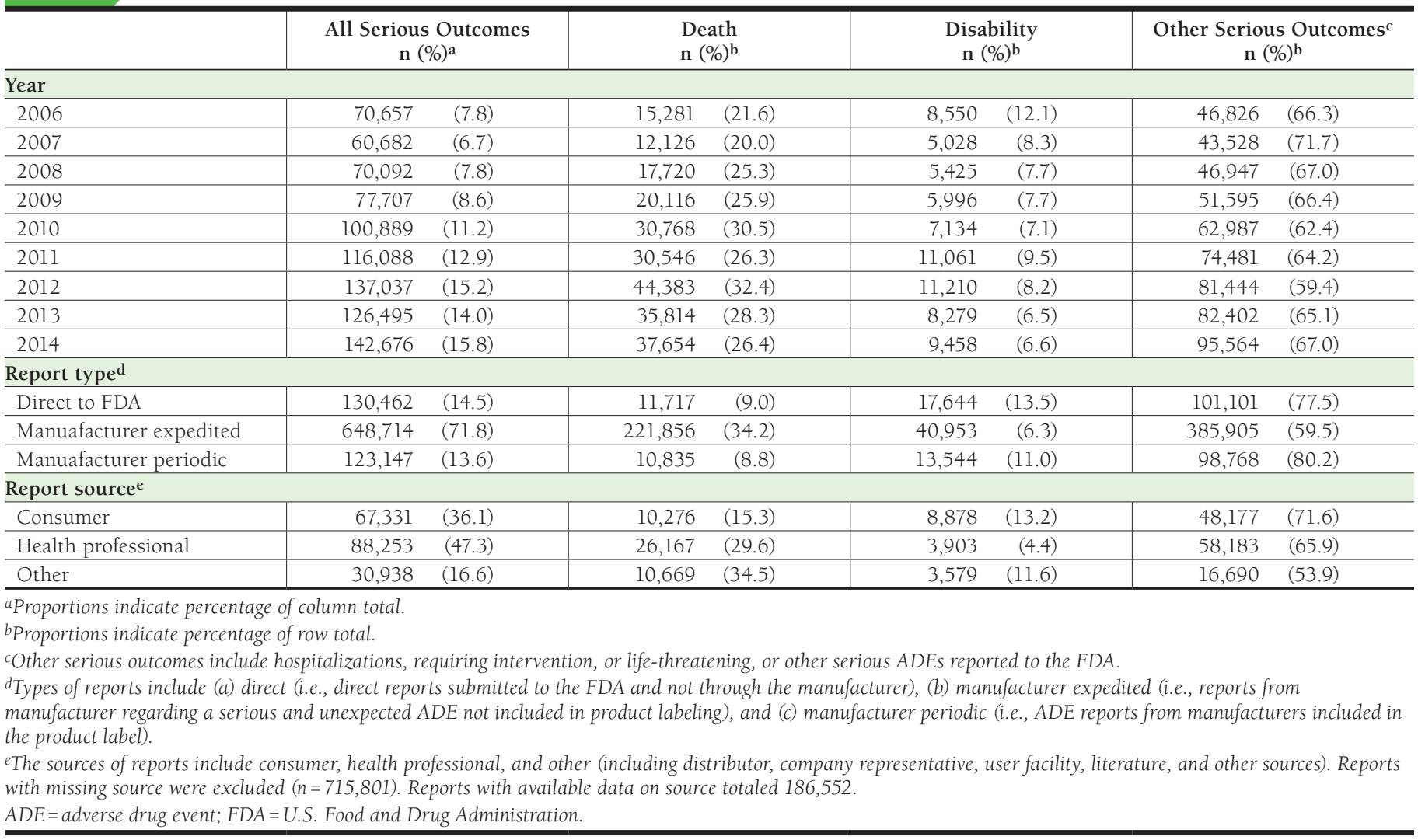

Although we attempted to mirror the analysis done by Moore et al., data cleaning or inclusion criteria applied in our study may have resulted in the slightly lower number of reports observed during these years. The transition from AERS to FAERS took place during August 2012; changes in organization of ADE reports or other structural changes might have resulted in the dip observed in 2013.

The differences could also be due to natural fluctuations in reporting rates over time or could be related to major public attention to ADEs that caused temporal changes in reporting rates. For example, Wysowski et al. (2005) reported fluctuations in the annual number of case reports in the AERS database from 1990 through 2002. ${ }^{13}$ Moore et al. speculated that part of the increase in serious ADE reports they observed might be due to release of pivotal research findings such as the 2002 reports from the Women's Health Initiative, which found risks associated with estrogen and progestin in postmenopausal women. ${ }^{14}$ Downswings in reporting trends may follow events such as these.

In addition to the release of research findings that may invoke ADE reporting, there may be public awareness campaigns or mandatory reporting programs that correspond to overall increases in reporting of ADEs. For example, REMS were authorized by the 2007 FDA Amendments Act, which requires mandatory risk management plans (e.g., continuous patient monitoring) for certain high-risk drugs. ${ }^{8}$ Ten drugs identified in our list of drugs with $>1,000$ reports in a given year were under a REMS program. Initiatives such as the REMS could have resulted in the increase in reporting of serious ADEs observed in our study following 2007. For instance, data published by the FDA show that the number of serious ADEs reports (including domestic and foreign reports) increased from 2006 to $2014 .^{3}$

One challenge is understanding whether increasing numbers of serious ADEs reports are a result of improved diligence in reporting (i.e., more events are reported) or more events are occurring. The potency and specificity of prescription drugs have been noted to be increasing over time, and it is possible that this is linked to a greater number of serious ADEs. ${ }^{15,16}$ Also, exposure to prescription medication may have escalated in the population as a result of an increase in the number of approved drugs, or an increase in use of drugs over time. ${ }^{7}$ Whatever the reason, increased efforts need to focus on monitoring ADEs to protect against possible negative outcomes associated with prescription drugs. 


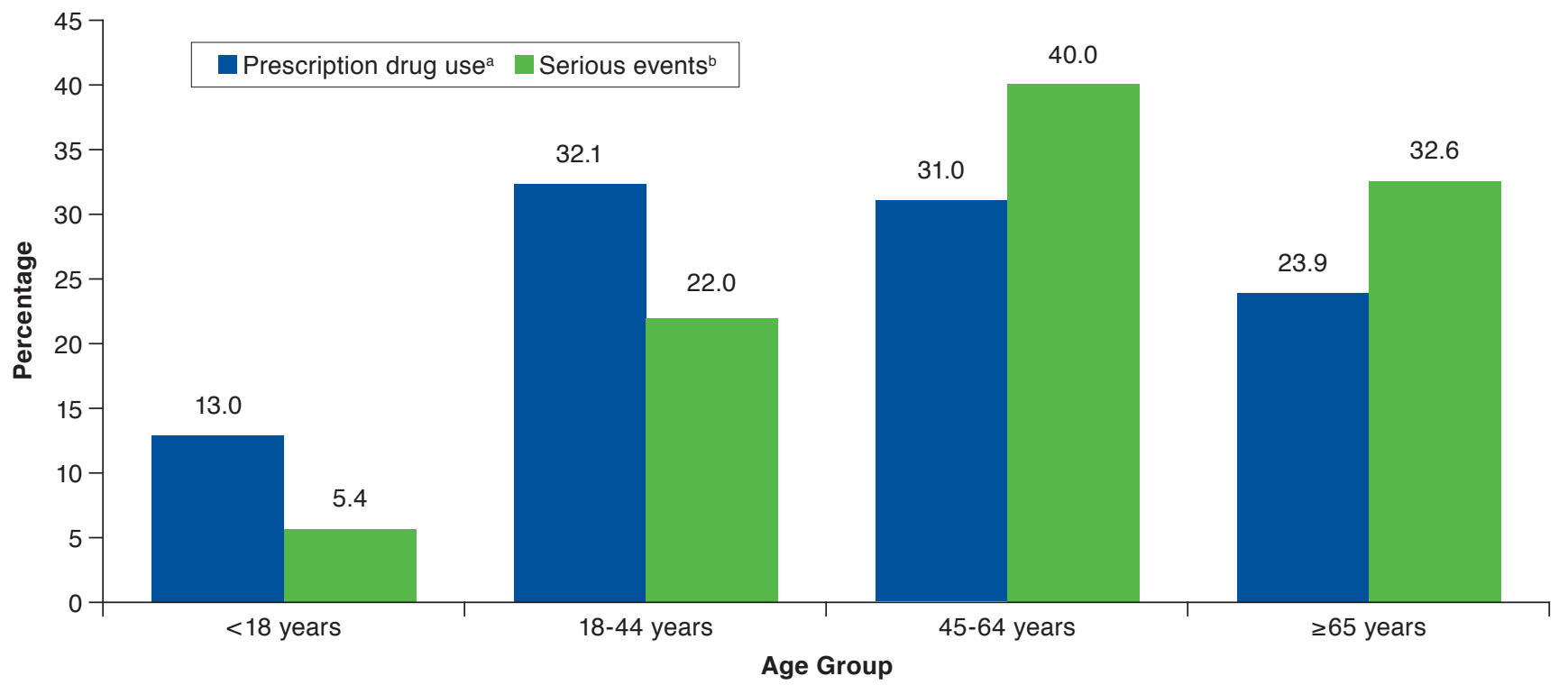

Note: This figure illustrates the distribution percentage of reported serious ADEs by age group in the United States during 2006-2014 with nonmissing data on age. ${ }^{a}$ Percent distribution of prescription drug use in the United States by age groups from 2007 to 2010 is from previously published data. ${ }^{b}$

a Reports with missing data on age were excluded $(n=268,038)$.

bProportion of prescription drug users within each age group for 2007-2010 was obtained from the National Center for Health Statistics document "Health United States, 2013: with special feature on prescription drugs." For percentage of population with at least 1 prescription drug in past 30 days see Table 92; for population within each age group, see Appendix Table $1 .^{7}$

$A D E=$ adverse drug event.

Expedited reports were the most common type of serious ADEs reports identified in our study, accounting for nearly $72 \%$ of reports. These reports are required to be submitted to the FDA by the drug manufacturer within a 15-day period of receiving a report for an ADE. Direct reports to the FDA by a consumer or health care professional constituted a much lower frequency (14.5\%).

This pattern is similar to that seen previously by Moore et al. (67.2\% expedited reports from manufacturers and 9.1\% direct reports). ${ }^{4}$ It is important to note that while expedited reporting is mandatory for manufacturers, direct reports to the FDA are voluntary ${ }^{17}$ which likely explains the gap between expedited and direct reporting.

Periodic reports constituted the smallest proportion of serious ADEs. Periodic reporting may be waived for drug manufacturers by the FDA for labeled and nonserious ADEs. ${ }^{18}$ Moreover, entering expedited and direct reports is prioritized by the FDA, ${ }^{19}$ and thus, periodic reports may not always be entered in FAERS.

Health care professionals are the most common initial source of serious ADEs reports. Consumers and other entities, including distributors, company representatives, user facilities, and literature also play a significant role in reporting serious
ADEs. However, it is likely that not all of the potential ADEs that can be reported from these sources are being reported. For instance, lack of patient-focused approaches in drug safety monitoring has been shown to be a significant barrier in capturing ADEs. ${ }^{20}$ Moreover, the general population may be less knowledgeable about recognizing an ADE or be less aware that they can report ADEs to the FDA. Nonetheless, ADE reporting by consumers and other entities adds significant value to the safety monitoring of drugs, and its promotion should be prioritized. $^{21}$

Data on the age distribution of serious ADEs from our study show that $40 \%$ of the reported ADEs occurred in patients aged 45-64 years, and over 32\% occurred in those aged $\geq 65$ years. A recent report by the CDC indicates that about $31 \%$ of the population of prescription drug users falls into the 45-64 year age group, and about 24\% falls into the $\geq 65$ year age group. ${ }^{7}$ Thus, disproportionately high proportions (approximately 73\%) of serious ADEs reports were among those aged $45-64$ years and $\geq 65$ years. There remains ambiguity on whether these data actually indicate a higher rate of ADEs among patients in this age group, or if ADEs among older patients are more likely to be reported. Nonetheless, these data highlight the disproportionate reporting and 


\begin{tabular}{|c|c|c|c|c|c|c|}
\hline \multirow[b]{2}{*}{ Drug Name } & \multirow[b]{2}{*}{ Drug Class } & \multirow[b]{2}{*}{ Rank } & \multirow[b]{2}{*}{$\begin{array}{l}\text { Number of } \\
\text { Reports }\end{array}$} & \multicolumn{3}{|c|}{ Report Source (\%) } \\
\hline & & & & $\begin{array}{c}\text { Health } \\
\text { Professional }\end{array}$ & Consumer & Other \\
\hline \multicolumn{7}{|l|}{ Death } \\
\hline Erlotinib & Antineoplastic & 1 & 11,451 & 95.6 & 2.3 & 2.0 \\
\hline Lenalidomide & Antineoplastic & 2 & 11,335 & 54.6 & 37.8 & 7.6 \\
\hline Rosiglitazone & Antidiabetic & 3 & 8,136 & 100.0 & 0.0 & 0.0 \\
\hline Bosentan & Antihypertensive & 4 & 8,036 & 39.2 & 0.3 & 60.5 \\
\hline Acetaminophen & Analgesic & 5 & 7,664 & 71.8 & 2.7 & 25.6 \\
\hline Bevacizumab & Antineoplastic & 6 & 6,052 & 88.4 & 1.6 & 10.0 \\
\hline Dianeal & Dialysis solution & 7 & 5,915 & 58.0 & 40.0 & 2.0 \\
\hline Ambrisentan & Antihypertensive & 8 & 4,468 & 100.0 & 0.0 & 0.0 \\
\hline Interferon beta la & Immunomodulator & 9 & 4,355 & 6.9 & 92.7 & 0.4 \\
\hline Alprazolam & Antianxiety & 10 & 4,080 & 70.7 & 0.6 & 28.8 \\
\hline \multicolumn{7}{|l|}{ Disability } \\
\hline Metoclopramide & Antiemetic & 1 & 9,932 & 0.5 & 68.3 & 17.9 \\
\hline Rofecoxib & Anti-inflammatory & 2 & 3,773 & 0.0 & 100.0 & 0.0 \\
\hline Paroxetine & Antidepressant & 3 & 3,275 & 31.4 & 31.4 & 1.2 \\
\hline Alendronate & Antiresorptive & 4 & 3,150 & 14.4 & 71.1 & 2.8 \\
\hline Quetiapine & Antipsychotic & 5 & 1,914 & 9.6 & 90.4 & 0.0 \\
\hline Levofloxacin & Antibiotic & 6 & 1,200 & 21.4 & 70.4 & 1.1 \\
\hline Lenalidomide & Antineoplastic & 7 & 1,122 & 59.1 & 30.9 & 6.6 \\
\hline Fluoxetine & Antidepressant & 8 & 1,083 & 22.6 & 51.6 & 0.7 \\
\hline Sertraline & Antidepressant & 9 & 911 & 19.3 & 59.6 & 1.3 \\
\hline Atorvastatin & Antihyperlipidemic & 10 & 739 & 7.8 & 86.9 & 1.1 \\
\hline \multicolumn{7}{|l|}{ Other serious outcomes } \\
\hline Interferon bla & Anti-inflammatory & 1 & 29,479 & 3.3 & 95.6 & 1.2 \\
\hline Rosiglitazone & Antidiabetic & 2 & 23,348 & 85.7 & 14.3 & 0.0 \\
\hline Dianeal (low calcium) & Dialysis solution & 3 & 18,874 & $\mathrm{NA}$ & NA & $\mathrm{NA}$ \\
\hline Teriparatide & Antiosteoporotic & 4 & 14,949 & 44.1 & 41.7 & 14.2 \\
\hline Etanercept & Immunomodulator & 5 & 14,445 & 95.5 & 4.5 & 0.0 \\
\hline Natalizumab & Immunomodulator & 6 & 14,080 & 49.2 & 50.8 & 0.1 \\
\hline Lenalidomide & Antineoplastic & 7 & 13,153 & 52.5 & 36.7 & 10.8 \\
\hline Dianeal & Dialysis solution & 8 & 12,726 & 51.2 & 47.3 & 1.5 \\
\hline Ambrisentan & Antihypertensive & 9 & 11,715 & 91.7 & 0.0 & 8.3 \\
\hline Dabigatran & Anticoagulant & 10 & 9,788 & 94.1 & 4.8 & 1.1 \\
\hline
\end{tabular}

aThe sources of reports include consumer, health professional, and other (including distributor, company representative, user facility, literature, and other sources).

Reports with missing source were excluded (death, $n=64,327$; disability, $n=19,397$; other serious outcomes, $n=140,601$ ).

$A D E=$ adverse drug event; FDA =U.S. Food and Drug Administration; NA = not available.

possible burden of serious ADEs among older patients using prescription drugs.

Drugs commonly identified as primary suspect in reports of serious ADEs were examined in further detail. Among these drugs, antineoplastic was one of the most frequently identified categories of drug classes among the top 10 drugs with death, disability, or other serious outcomes. This finding is not surprising, because antineoplastics are known to have serious adverse effects. It is also possible that the reported deaths and disabilities are confounded by the nature of the disease being treated (i.e., cancer). Acetaminophen was ranked fifth in the list of the top 10 drugs with fatal outcomes. ADEs due to opioid analgesics have received public attention in recent years. ${ }^{22,23}$ The prevalence of off-label use, abuse, drug-drug interactions, and over-thecounter access to analgesics could be driving the high number of serious ADEs with agents in this drug class. ${ }^{24-26}$ It is noteworthy that the top 10 drugs identified in the outcome categories (i.e., death, disability, and other serious outcomes) constituted for $>25 \%$ of the total number of reports in the respective category.

In addition to drugs involved in specific types of serious ADEs, we identified a list of 39 drugs with more than 1,000 reports of any serious ADEs during a given year. Of these, 2 drugs were withdrawn from the market (rofecoxib and parecoxib). Litigation and public awareness may have contributed to the high number of reports of ADEs for these drugs. For instance, according to a 2006 report in USA Today, about 14,000 lawsuits were filed against 
Serious Adverse Drug Events Reported to the FDA: Analysis of the FDA Adverse Event Reporting System 2006-2014 Database

TABLE 3 Drugs with More Than 1,000 Reports of Serious ADEs in Any Year in FAERS, 2006-2014

\begin{tabular}{|c|c|c|c|c|c|c|c|c|c|c|}
\hline Drug Name & Total & 2006 & 2007 & 2008 & 2009 & 2010 & 2011 & 2012 & 2013 & 2014 \\
\hline \multicolumn{11}{|c|}{ Drugs withdrawn from the market } \\
\hline Rofecoxib & 12,744 & 12,692 & 10 & 15 & 3 & 1 & 8 & 1 & 11 & 3 \\
\hline Parecoxib & 3,353 & 1,094 & 1,730 & 279 & 37 & 198 & 7 & 4 & 4 & 0 \\
\hline \multicolumn{11}{|c|}{ Drugs with a REMS program } \\
\hline Lenalidomide & 22,549 & 620 & 1,089 & 1,617 & 1,675 & 2,185 & 2,923 & 4,983 & 3,837 & 3,620 \\
\hline Ambrisentan & 16,184 & 0 & 38 & 436 & 721 & 1,332 & 2,323 & 2,921 & 3,847 & 4,566 \\
\hline Natalizumaba $^{a}$ & 15,261 & 2 & 32 & 936 & 1,689 & 2,344 & 2,652 & 2,320 & 2,982 & 2,304 \\
\hline Teriparatide $^{\mathrm{a}}$ & 8,388 & 951 & 814 & 612 & 925 & 1,473 & 1,566 & 1,319 & 598 & 130 \\
\hline Varenicline & 5,096 & 27 & 545 & 927 & 371 & 1,308 & 937 & 603 & 207 & 171 \\
\hline Thalidomide & 3,527 & 405 & 281 & 1,271 & 257 & 210 & 181 & 160 & 309 & 453 \\
\hline Olanzapine & 3,401 & 1,266 & 938 & 177 & 366 & 270 & 188 & 119 & 42 & 35 \\
\hline Denosumab $^{a}$ & 2,789 & 0 & 0 & 0 & 0 & 17 & 118 & 278 & 764 & 1,612 \\
\hline Eculizumaba & 2,450 & 0 & 1 & 7 & 189 & 211 & 201 & 185 & 223 & 1,433 \\
\hline Pomalidomide & 1,746 & 0 & 0 & 0 & 0 & 0 & 0 & 0 & 576 & 1,170 \\
\hline \multicolumn{11}{|c|}{ Biologic or specialty drugs } \\
\hline Interferon bla & 34,152 & 3,086 & 3,384 & 4,341 & 4,347 & 4,212 & 3,937 & 3,552 & 4,098 & 3,195 \\
\hline Etanercept & 16,221 & 770 & 1,225 & 1,255 & 1,236 & 1,435 & 1,498 & 2,232 & 4,060 & 2,510 \\
\hline Bosentan & 15,805 & 81 & 49 & 129 & 163 & 4,717 & 2,602 & 2,801 & 2,588 & 2,675 \\
\hline Erlotinib & 9,971 & 157 & 94 & 99 & 125 & 164 & 180 & 8,413 & 460 & 279 \\
\hline Adalimumab & 7,018 & 333 & 509 & 850 & 981 & 936 & 1,374 & 1,349 & 301 & 385 \\
\hline Infliximab & 6,449 & 703 & 441 & 475 & 537 & 586 & 920 & 1,064 & 985 & 738 \\
\hline Alendronate & 6,023 & 361 & 367 & 511 & 453 & 589 & 660 & 1,385 & 893 & 804 \\
\hline Bevacizumab & 5,919 & 453 & 316 & 278 & 161 & 134 & 199 & 2,975 & 680 & 723 \\
\hline Exenatide & 4,342 & 495 & 597 & 606 & 1,030 & 584 & 423 & 176 & 162 & 269 \\
\hline Treprostinil & 3,659 & 61 & 33 & 8 & 8 & 433 & 1,260 & 203 & 289 & 1,364 \\
\hline Capecitabine & 3,251 & 133 & 170 & 178 & 143 & 140 & 110 & 1,281 & 649 & 447 \\
\hline Dimethyl fumarate & 2,496 & 0 & 0 & 0 & 0 & 0 & 0 & 0 & 618 & 1,878 \\
\hline Enzalutamide & 1,929 & 0 & 0 & 0 & 0 & 0 & 0 & 51 & 759 & 1,119 \\
\hline \multicolumn{11}{|l|}{ Other drugs } \\
\hline Rosiglitazone & 29,270 & 84 & 294 & 718 & 2,349 & 7,668 & 6,899 & 4,188 & 3,518 & 3,552 \\
\hline Dianeal & 16,751 & 124 & 290 & 1,153 & 1,577 & 3,453 & 6,296 & 3,858 & 0 & 0 \\
\hline Dianeal (low calcium) & 14,953 & 0 & 0 & 0 & 0 & 0 & 0 & 1,464 & 8,432 & 5,057 \\
\hline Dabigatran & 11,468 & 0 & 0 & 0 & 1 & 136 & 2,763 & 2,591 & 2,322 & 3,655 \\
\hline Metoclopramide & 10,108 & 16 & 16 & 24 & 50 & 488 & 5,350 & 3,809 & 339 & 16 \\
\hline Quetiapine & 8,153 & 1,603 & 1,548 & 475 & 1,647 & 1,229 & 884 & 498 & 174 & 95 \\
\hline Deferasirox & 6,777 & 125 & 96 & 111 & 1,513 & 1,665 & 1,702 & 1,229 & 141 & 195 \\
\hline Dianeal (with dextrose) & 5,638 & 0 & 0 & 0 & 0 & 0 & 0 & 0 & 0 & 5,638 \\
\hline Baclofen & 4,883 & 106 & 699 & 1,174 & 1,080 & 795 & 494 & 179 & 143 & 213 \\
\hline Esomeprazole & 4,185 & 64 & 89 & 138 & 250 & 206 & 1,094 & 984 & 733 & 627 \\
\hline Rivaroxaban & 3,999 & 0 & 0 & 0 & 0 & 0 & 50 & 712 & 1,503 & 1,734 \\
\hline Alprazolam & 3,738 & 80 & 26 & 231 & 229 & 482 & 397 & 450 & 1,056 & 787 \\
\hline Clozapine & 3,232 & 1,621 & 66 & 64 & 69 & 271 & 424 & 395 & 204 & 118 \\
\hline Dianeal (ultrabag) & 1,760 & 0 & 0 & 0 & 0 & 0 & 0 & 233 & 1,078 & 449 \\
\hline
\end{tabular}

rofecoxib. ${ }^{27}$ During the same year, more than 12,692 serious ADEs with rofecoxib as primary suspect were reported in FAERS. The withdrawal of rofecoxib toward the end of 2004, and subsequent litigation, may have contributed to the high number of ADE reports we observed early in our study period. Moreover, 10 of these 39 drugs had a REMS program. Mandatory patient moni- toring under REMS may have contributed to higher ADE reports for these drugs.

It is interesting to note that a significant number of biologic and specialty drugs were on this list. In a recent network meta-analysis, biologic drugs were found to be associated with a $28 \%$ higher risk of ADEs compared with a nonbiologic 
control group. ${ }^{28}$ Biologic and specialty drugs also are known for their high acquisition costs. The presence of these drugs in a significant number of serious ADEs indicates that a patient's economic burden may be even higher if costs of treating serious ADEs from these drugs are taken in consideration. Future studies should investigate the costs of treating ADEs in addition to the acquisition costs of these drugs to understand their true economic burden on the public.

\section{Limitations}

Our study has several limitations. First, the study is descriptive in nature. ADEs in FAERS are spontaneously reported, so the true incidence of ADEs cannot be determined using these data. An observational data source that is comprehensive and includes information on the use of prescription drugs and the resulting ADEs would allow determination of ADE rates in the real world. Availability of such data will also help us understand the reasons for disproportionate burden of ADEs by patient demographic. For instance, age standardization would help to evaluate whether observed trends can be explained by an aging population. However, observational data of such nature are currently unavailable and, given this data constraint, FAERS provides a unique opportunity for descriptive ADE research.

Second, uncertainty regarding the drug's role in a reported $\mathrm{ADE}$ exists due to the voluntary nature of reports. The role of other drugs that were used concomitantly with the primary suspect drug when the ADE occurred cannot be completely ruled out. Therefore, causal inferences between drug and ADE cannot be drawn from the data.

Third, ADE reporting may be influenced by several external factors, such as duration the drug is in the market, litigation, and advertising or other media attention, and these factors cannot be addressed in our analyses. Finally, reporting trends of ADEs have been shown to vary over time., ${ }^{4,13}$ Whether these variations in trends accurately reflect the actual variations in the occurrence of ADEs or are a result of changes in reporting patterns cannot be ascertained. The results of our analysis must be interpreted in the context of these limitations.

\section{Conclusions}

Prescription drugs are a necessary component of the treatment of diseases and disorders, but the occurrence of serious ADEs can cause the benefits of these drugs to be outweighed by the harm. Our study of FAERS indicates that reports of serious ADEs increased from 2006 through 2014. Biologic and specialty drugs had a substantial number of serious ADE reports. FAERS is a key component of postmarketing surveillance and is a valuable resource that supports ongoing efforts to understand the public health burden of ADEs.

\section{Authors}

KALYANI B. SONAWANE, PhD, Department of Health Services Research, Management and Policy, University of Florida, Gainesville. NING CHENG, MS, and RICHARD A. HANSEN, PhD, Department of Health Outcomes Research and Policy, Auburn University, Auburn, Alabama.

AUTHOR CORRESPONDENCE: Kalyani B. Sonawane, PhD, Department of Health Services Research, Management and Policy, University of Florida, 1225 Center Dr., HPNP 3118, Gainesville, FL 32611. Tel.: 352.273.6074; E-mail: ksonawane@phhp.ufl.edu.

\section{DISCLOSURES}

No outside funding supported this study. Hansen has received consulting fees from and has provided expert testimony for Daichii Sankyo and Takeda. The other authors have nothing to disclose.

\section{ACKNOWLEDGMENTS}

The authors acknowledge Sarah K. Dutcher, PhD, for her valuable insights on this manuscript, and Raj Desai, MS, for his assistance in preparing this manuscript.

\section{REFERENCES}

1. U.S. Food and Drug Administration. Summary of NDA approvals \& receipts, 1938 to the present. 2015. Updated February 2018. Available at: https://www.fda.gov/aboutfda/whatwedo/history/productregulation/ ucm2006085.htm. Accessed May 21, 2018.

2. U.S. Food and Drug Administration. Potential signals of serious risks/ new safety information identified from the FDA Adverse Event Reporting System (FAERS). 2015. Updated April 2018. Available at: http://www. fda.gov/Drugs/GuidanceComplianceRegulatoryInformation/Surveillance/ AdverseDrugEffects/UCM082196. Accessed May 21, 2018.

3. U.S. Food and Drug Administration. FAERS reporting by patient outcomes by year. November 2015. Available at: http://www.fda.gov/ Drugs/GuidanceComplianceRegulatoryInformation/Surveillance/ AdverseDrugEffects/ucm070461.htm. Accessed May 21, 2018.

4. Moore TJ, Cohen MR, Furberg CD. Serious adverse drug events reported to the Food and Drug Administration, 1998-2005. Arch Intern Med. 2007;167(16):1752-59.

5. U.S. Food and Drug Administration. FDA Adverse Event Reporting System (FAERS): latest quarterly data files. 2015. Updated April 2018. Available at: http://www.fda.gov/Drugs/ GuidanceComplianceRegulatoryInformation/Surveillance/ AdverseDrugEffects/ucm082193.htm. Accessed May 21, 2018.

6. U.S. Government Printing Office. Electronic code of federal regulations Title 21-food and drugs. 21 CFR (Parts 300-499) (2015). Available at: http://www.ecfr.gov/cgi-bin/text-idx?SID=0b3aadf46847c8c4ea65887c06lfa 89c\&mc=true\&tpl=ibr/21V5.tpl. Accessed May 21, 2018.

7. National Center for Health Statistics. Health, United States, 2013: with special feature on prescription drugs. DHHS Publication no. 2014-1232. May 2014. Available at: https://www.cdc.gov/nchs/data/hus/hus13.pdf. Accessed May 21, 2018.

8. U.S. Food and Drug Administration. Postmarket drug safety information for patients and providers. REMS@FDA: approved risk evaluation and mitigation strategies (REMS). 2017. Available at: https://www.fda.gov/Drugs/ DrugSafety/PostmarketDrugSafetyInformationforPatientsandProviders/ default.htm. Accessed May 24, 2018. 
9. Nurmohamed MT, Dijkmans BA. Efficacy, tolerability and cost effectiveness of disease-modifying antirheumatic drugs and biologic agents in rheumatoid arthritis. Drugs. 2005;65(5):661-94.

10. Gisondi P, Girolomoni G. Biologic therapies in psoriasis: a new therapeutic approach. Autoimmun Rev. 2007;6(8):515-19.

11. UnitedHealth Center for Health Reform \& Modernization. The growth of specialty pharmacy. Issue brief. April 2015. Available at: http://www.unitedhealthgroup.com/ /media/UHG/PDF/2014/UNH-The-Growth-Of-SpecialtyPharmacy.ashx. Accessed May 21, 2018.

12. BlueCross BlueShield of North Carolina. Current specialty medication list. 2015. Updated April 2018. Available at: https://www.bcbsnc.com/assets/ services/public/pdfs/formulary/specialty-network/specialty-drug-list.pdf. Accessed May 21, 2018.

13. Wysowski DK, Swartz L. Adverse drug event surveillance and drug withdrawals in the United States, 1969-2002: the importance of reporting suspected reactions. Arch Intern Med. 2005;165(12):1363-69.

14. Rossouw JE, Anderson GL, Prentice RL, et al. Risks and benefits of estrogen plus progestin in healthy postmenopausal women: principal results from the Women's Health Initiative randomized controlled trial. JAMA. 2002;288(3):321-33.

15. Corrao G, Soranna D, Casula M, Merlino L, Porcellini MG, Catapano AL. High-potency statins increase the risk of acute kidney injury: evidence from a large population-based study. Atherosclerosis. 2014;234(1):224-29.

16. Bischoff E. Potency, selectivity, and consequences of nonselectivity of PDE inhibition. Int J Impot Res. 2004;16 Suppl 1:S11-14.

17. U.S. Food and Drug Administration. Reporting serious problems to FDA. 2015. Updated March 2018. Available at: http://www.fda.gov/Safety/ MedWatch/HowToReport/. Accessed May 21, 2018.

18. Elliott WJ, Plauschinat CA, Skrepnek GH, Gause D. Persistence, adherence, and risk of discontinuation associated with commonly prescribed antihypertensive drug monotherapies. J Am Board Fam Med. 2007;20(1):72-80.
19. U.S. Food and Drug Administration. Reports received and reports entered into FAERS by year. 2013. Updated November 2015. Available at: http://www.fda.gov/Drugs/GuidanceComplianceRegulatoryInformation/ Surveillance/AdverseDrugEffects/ucm070434.htm. Accessed May 21, 2018.

20. Basch E. The missing voice of patients in drug-safety reporting. N Eng J Med. 2010;362(10):865-69.

21. Blenkinsopp A, Wilkie P, Wang M, Routledge PA. Patient reporting of suspected adverse drug reactions: a review of published literature and international experience. Br J Clin Pharmacol. 2007;63(2):148-56.

22. Joint Commission on Accreditation of Healthcare Organizations. Safe use of opioids in hospitals. Sentinel event alert. August 8, 2012. Available at: https://www.jointcommission.org/assets/1/18/SEA_49_opioids_8_2_12_ final.pdf. Accessed May 21, 2018.

23. Centers for Disease Control and Prevention. Medication safety program: program focus and activities. 2015. Updated June 2017. Available at: http:// www.cdc.gov/MedicationSafety/program_focus_activities.html. Accessed May 21, 2018.

24. Phan H, Leder M, Fishley M, Moeller M, Nahata M. Off-label and unlicensed medication use and associated adverse drug events in a pediatric emergency department. Pediatr Emerg Care. 2010;26(6):424-30.

25. Abbott FV, Fraser MI. Use and abuse of over-the-counter analgesic agents. J Psychiatry Neurosci. 1998;23(1):13-34.

26. Wright A, Feblowitz J, Phansalkar S, et al. Preventability of adverse drug events involving multiple drugs using publicly available clinical decision support tools. Am J Health Syst Pharm. 2012;69(3):221-27.

27. Schmit J. More drugs get slapped with lawsuits. USA Today. August 23 , 2006. Available at: http://usatoday30.usatoday.com/money/industries/health/ drugs/2006-08-23-drug-lawsuits-usat_x.htm. Accessed May 21, 2018.

28. Singh JA, Wells GA, Christensen R, et al. Adverse effects of biologics: a network meta-analysis and Cochrane overview. Cochrane Database of Systematic Reviews. 2011(2):CD008794. 